\title{
Challenges in the Assessment of Children with Developmental Coordination Disorder (Dcd)
}

\author{
U Ganapathy sankar ${ }^{1}$ and Monisha $\mathbf{R}^{* 2}$ \\ ${ }^{1}$ Professor and dean, SRM Institute of science and technology, India \\ ${ }^{2}$ Assistant professor, SRM Institute of science and technology, India
}

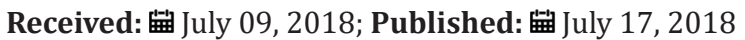

*Corresponding author: Monisha R, Assistant Professor, SRM College of Physiotherapy, SRM Institute of Science and Technology, Chennai, India

\begin{abstract}
Children with DCD have difficulty in organizing and sequencing of motor events. Instead of having higher IQ of greater than 70\% these children face difficulties in reading and writing. All other activities are daily living is a biggest challenge for these children to be done without assistance from their caregivers. Without proper evaluation of these children as "DCD", they have been diagnosed as a continuum of cerebral palsy and all the caregivers of children with DCD waste their time and money on the inappropriate diagnosis and treatment interventions.
\end{abstract}

\section{Introduction}

DCD is a neurodevelopmental disorder which has been misdiagnosed as cerebral palsy in the early years of child's development, these children have poor participation in physical activities and they experiences difficulty in performing all the activity of daily living. Early manifestation of children with DCD includes delay in acquisition of motor milestone which is identified by poor standing and walking balance, poor performance in all the activity of daily living and with their untidy handwriting because of poor prehension handling [1]. World Health Organization and American Psychiatric Association has given their unique definition in the identification and classification of these children with Developmental coordination Disorder. However, both the definition on DCD overlaps and there exist a maximum number of difficulties in classification and identification of children with DCD [2].

When the historical background of the Developmental Coordination Disorder has been analyzed, there exist a difficulty in identifying children with DCD because of the wide variation in the terminology. These children have been referred as Clumsy, Awkward, motorically poor, Apraxia and developmental disability. Because of the variation in terminology, these children were classified with poor knowledge of the condition [3]. Dr U. Ganapathy Sankar and Saritha conducted a study on Prevalence of Developmental Coordination Disorder at Kattankulathur, Tamilnadu in 2011 and Concluded that the prevalence rate was $1.37 \%$ with the age group of 5-10 years at Kattankulathur, Tamilnadu [4]. Dr U Ganapathy Sankar conducted a study on Prevalence of Developmental Coordination Disorder at Kattupakkam, Tamilnadu in 2018 [5] and Concluded that the prevalence rate is $3.22 \%$, When gender differences has been taken into account, more boys than girls (2:1) being diagnosed with DCD. However, Prevalence of DCD is directly related to the manner in which assessment tool is employed. There is still much debate and difficulty in estimating the prevalence rate of DCD in Indian population.

\section{Review}

When the prevalence estimate of DCD has been analyzed, there is an error which has been left un-noticed, previous researchers have given the prevalence estimate value, but none of them have used a gold standard assessment measure in estimating the prevalence rate, Bruininks Oseretsky Test of Motor Proficiency, Developmental Coordination Disorder Questionnaire, Movement Assessment Battery for Children are the most commonly used assessment measures in the identification of children with DCD. But they exist a wide variation in the internal consistency of the assessments that is used formally in the identification of children. When the etiology is taken into account there is a debate which is worth considering, researchers have highlighted that it is a continuum of cerebral palsy and prenatal, perinatal and neonatal insults can result in DCD which is highlighted by few researchers in past. Damage at the cellular level can result in DCD, these are the various hypothesis stated by previous researchers and everything ends with a challenge in the diagnosis of these children with developmental coordination disorder.

\section{Conclusion}

There is an inconsistency among motor tests which is used in identification of children with DCD and all researches in DCD ends in a hypothesis. There is no standardized method can accurately identify DCD children, there are still few therapists 
classify these children as cerebral palsy without proper knowledge about the coordination difficulty. Further research is needed in the development of reliable assessment method and there is an increasing demand for the therapeutic treatment protocol regimen for children with DCD in Indian Population.

\section{References}

1. American Psychiatric Association (2013) Diagnostic and statistical manual of mental disorders ( $5^{\text {th }}$ Edn.). Washington DC, USA.

2. Beery KE, Beery NA (2004) Beery VMI: The Beery-Buktenica developmental test of visual-motor integration ( $5^{\text {th }}$ Edn.). Minneapolis MN: Pearson, USA.

ISSN: 2574-1241

DOI: 10.26717/BJSTR.2018.06.001424

Monisha R. Biomed J Sci \& Tech Res

(C) This work is licensed under Creative

Submission Link: https://biomedres.us/submit-manuscript.php
3. Ganapathy Sankar U, Saritha S (2011) A study of prevalence of Developmental Coordination Disorder (DCD) at Kattankulathur, Chennai. Indian Journal of Physiotherapy and occupational therapy Jan5(1): 63-65.

4. U Ganapathy Sankar (2018) The Prevalence of Developmental Coordination Disorder at Kattupakkam, Tamilnadu. IOSR Journal of Pharmacy 8(2): 49-52.

5. Coltheart M, Rastle K, Perry C, Langdon R, Ziegler JC (2001) DRC: A dual route cascaded model of visual word recognition and reading aloud. Psychological Review 108(1): 204-256.

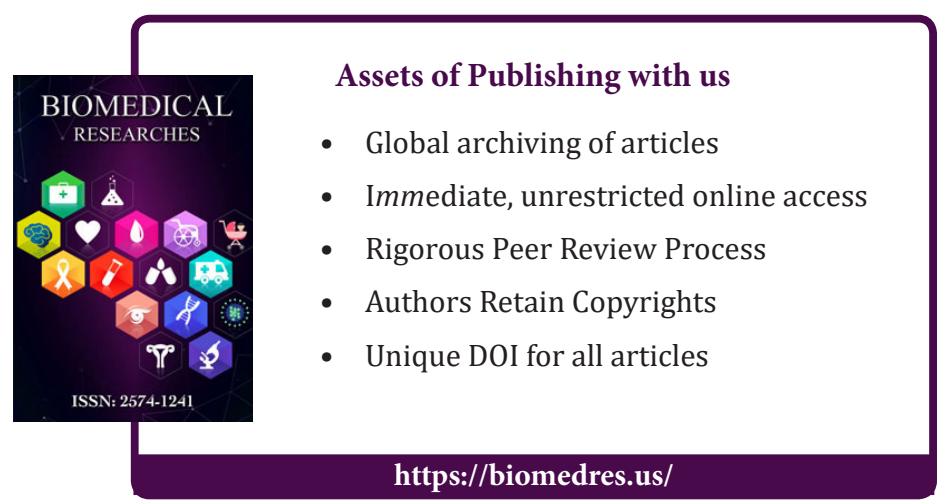

